\title{
Systematic analysis of synergistic proteome modulations in a drug combination of cisplatin and MLN4924†
}

\author{
Dominik Andre Megger, (D) *ab Shadi Abou-Eid, ${ }^{\text {ac }}$ Birgit Zülch $^{a}$ and Barbara Sitek ${ }^{a}$
}

\begin{abstract}
Chemotherapeutic treatment regimens often take advantage of synergistic effects of drug combinations. Anticipating that synergistic effects on the cell biological level likely manifest on the proteome level, the analysis of proteome modulations represents an appropriate strategy to study drug combinations on a molecular level. More specifically, the detection of single proteins exhibiting synergistic abundance changes could be helpful to shed light on key molecules, which contribute in mechanisms facilitating the synergistic interaction and therefore represent potential targets for specific therapeutic approaches. In the reported study we aimed to provide evidence for this assumption and investigated the drug combination of cisplatin and the neddylation inhibitor MLN4924 in HCT-116 cells via cell biological analyses and mass spectrometry-based quantitative proteomics. From 1789 proteins quantified with two unique peptides, activated RNA polymerase II transcriptional coactivator p15 (SUB1) was highlighted as the most synergistically regulated protein using a synergistic scoring approach. Western blotting and analyses of cellular processes associated with this protein (DNA damage, oxidative stress and apoptosis) revealed supporting evidence for the synergistic regulation. Whereas the distinct role of SUB1 in the investigated drug combination needs to be elucidated in future studies, the presented results demonstrated the benefit and feasibility of synergistic scoring of proteome alterations to highlight proteins that likely contribute to the underlying molecular mechanisms of synergistic effects. Data are available via ProteomeXchange with identifier PXD009185.
\end{abstract}

\section{Introduction}

Since the discovery of its applicability as an antitumor agent more than half a century ago, ${ }^{1}$ cisplatin has been utilized in chemotherapeutic treatments of various malignant diseases including bladder, head and neck, ovarian, testicular, breast, brain and lung cancers. ${ }^{2,3}$ However, the establishment of resistance and therefore the relapse of patients with cisplatinresistant diseases represents a major problem of platinum-based chemotherapy. Hence, cisplatin is commonly administered in combination with other drugs such as doxorubicin, gemcitabine,

${ }^{a}$ Medizinisches Proteom-Center, Ruhr-Universität Bochum, Universitätsstr. 150, 44801 Bochum, Germany. E-mail: dominik.megger@rub.de

${ }^{b}$ Institute for Virology, University Hospital Essen, University Duisburg-Essen, Virchowstraße 179, 45147 Essen, Germany

${ }^{c}$ Department of Biology, University of Fribourg, Chemin du Musée 10, 1700 Fribourg, Switzerland

$\dagger$ Electronic supplementary information (ESI) available: Result of synergy analysis using SynergyFinder web application. Supplementary tables contain lists of all quantified proteins (Table S1), proteins exclusively regulated upon combined treatment and calculated synergistic scores (Table S2) as well as an overview of LC-MS/MS run identifiers (Table S3). See DOI: 10.1039/c8mo00115d anvirzel, paclitaxel or 5 -fluorouracil. ${ }^{3}$ Furthermore, combination therapies also aim to minimize the side effects of single drug therapies since the doses of individual compounds can be remarkably reduced in the case of a synergistic drug combination. Finding applicable combinations for disease-specific treatment regimens represents a labor-intensive process that mostly starts with the screening of various drug combinations in cell culture models and the assessment of antagonistic, additive and synergistic effects. Such screenings are then often followed by extensive functional studies to elucidate underlying molecular mechanisms of synergistic interactions.

In addition to drug-induced DNA damage, targeting cellular protein homeostasis represents an alternative strategy to treat various types of cancer. Bortezomib, for example, is a proteasome inhibitor which is used for the treatment of multiple myeloma and mantle cell lymphoma. ${ }^{4,5}$ A more recent example of a drug targeting cellular protein homeostasis is MLN4924, also known as pevonedistat. This drug is an adenosine sulfamate analog that selectively inhibits NEDD8-activating enzyme (NAE), an enzyme playing a key role in the so called neddylation process. ${ }^{6,7}$ By disrupting the activity of NAE, MLN4924 blocks the neddylation of cullin-RING ligases (CRLs). Since functionalization with NEDD8 

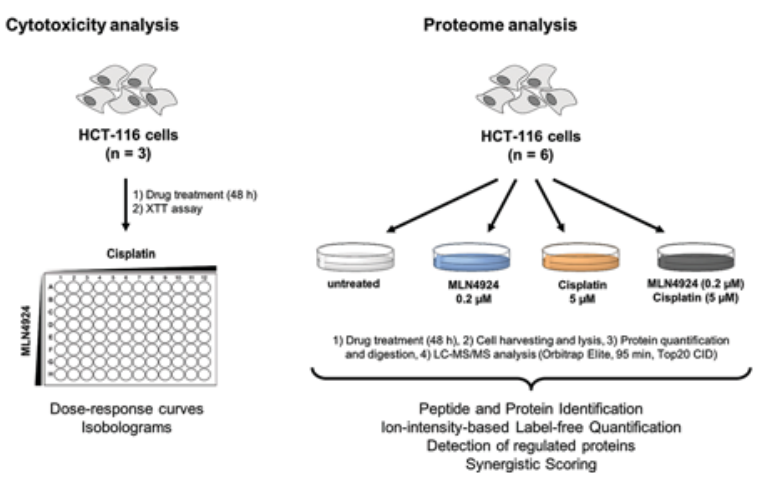

Fig. 1 Overview of the study design and experimental workflow.

is necessary for the activation of CRLs, MLN4924 thereby interferes with the ubiquitin-proteasome system (UPS). As a single agent, MLN4924 has been shown to be effective against various malignancies $^{8}$ and also applicable for antiviral treatments. ${ }^{9}$ Furthermore, in various independent studies, MLN4924 has been shown to sensitize multiple types of cancer cells towards DNA-damaging drugs such as cisplatin..$^{10-15}$

In the presented study, we investigated the synergistic drug combination of cisplatin and MLN4924 in the human colon carcinoma cell line HCT-116 (Fig. 1). In a first step, we aimed to reproduce the reported synergy ${ }^{15}$ on a cell biological level by cytotoxicity analyses of drug combinations across a wide concentration range of individual compounds. In a subsequent quantitative proteome analysis of differentially treated cells, we then wanted to address the question of whether synergistic effects on the cell biological level also manifest on the proteome level in terms of synergistic protein regulations. Therefore, we applied an ion-intensity-based label-free quantitative approach, which we have proven in the past to be suitable to study particular treatment responses of cells on the proteome level. ${ }^{9,16,17}$ Furthermore, for the first time, we implemented a synergistic scoring in our data analysis workflow to obtain a quantitative measure of synergy of individual protein regulations in addition to the mostly conducted accession-based comparison of regulated proteins by Venn diagrams as reported in previous studies using quantitative approaches such as iTRAQ ${ }^{18}$ spectral counting ${ }^{19}$ and 2D-DIGE. ${ }^{20}$ This unique approach allowed us to highlight synergistically regulated proteins and to differentiate these from rather additive regulations. Finally, we analyzed and discussed if and to what extent the observed synergistic protein regulations coincide with and therefore support proposed modes of action of the investigated drug combination (i.e. increased DNA damage and oxidative stress).

\section{Results and discussion}

\section{Cytotoxicity analyses}

The cytotoxic properties of MLN4924 and cisplatin as well as various combinations of these compounds were investigated in the colorectal carcinoma cell line HCT-116. To assess dosedependent cytotoxic effects of both individual compounds and respective drug combinations, cells were treated for $48 \mathrm{~h}$ with different concentrations of the compounds in nano- and micromolar ranges. Using the XTT assay, cell viability was determined in a 96-well format for each condition relative to a carrier control (1\% DMSO). Based on the data acquired in five individual experiments, dose-response curves were generated for both compounds (Fig. 2A and $\mathrm{B}$ ) and $\mathrm{EC}_{50}$ values for MLN4924 $(0.41 \pm 0.01 \mu \mathrm{M})$ and cisplatin $(14.12 \pm 0.04 \mu \mathrm{M})$ were calculated. These values are in good agreement with reported $\mathrm{EC}_{50}$ values for both drugs and the respective cell line (cisplatin: $13 \mu \mathrm{M},{ }^{15}$ MLN4924: $0.26 \mu \mathrm{M}^{21}$ ).

In drug combinations, the dose-response curves of both drugs are shifted to lower concentrations indicating decreased $\mathrm{EC}_{50}$ values and consequently a sensitization of the cancer cells towards cisplatin by MLN4924 and vice versa. For example, at concentrations of $0.25 \mu \mathrm{M}$ MLN4924 and $8.3 \mu \mathrm{M}$ cisplatin, respectively, the individual drugs induce only moderate reduction of cell viability. However, in combination, the number of vital cancer cells is tremendously dropped (Fig. 2C). In the following, this combination effect was further investigated to decipher whether it is of synergistic or additive nature. Therefore, MLN4924 and cisplatin concentrations of drug combinations leading to a $50 \%$ reduction of cell viability were plotted in an isobologram (Fig. 2D). The fact that the data points for $\mathrm{EC}_{50}$ values of drug combinations are located below the isobole of additivity (dashed line) indicates a synergistic interaction. ${ }^{22}$ The observed concave curve nicely matches previously reported data acquired for the same cell line and drug combination. ${ }^{15}$ Furthermore, data analysis using the


Fig. 2 Results of cell viability studies. (A) and (B) show dose-response curves of single agents and one selected drug combination. The cooperative effect of both drugs is indicated by shifts of the sigmoidal curves towards lower drug concentrations. (C) shows cell viabilities of selected concentrations (in $\mu \mathrm{M}$ ) of single agents and a drug combination ( $* * *=p<0.001$, Mann-Whitney-test). Values correspond to averaged cell viabilities (normalized to carrier control) assessed in three independent experiments \pm one standard deviation. (D) shows the results of an isobologram analysis. The dashed line shows the isobole of additivity and the solid line is a nonlinear curve fit of drug combinations leading to a $50 \%$ reduction of cell viability $\left(R^{2}=0.99\right)$. 
SynergyFinder web application ${ }^{23}$ supports the assumed synergy (see: $\mathrm{ESI} \dagger$ ).

\section{General results of the proteome analysis}

To analyze how far the observed synergy is displayed on the proteome level, alterations of the proteomes of cells treated with MLN4924 $(0.2 \mu \mathrm{M})$, cisplatin $(5 \mu \mathrm{M})$ or a combination of both drugs at the respective concentrations were quantitatively monitored in comparison to untreated cells. Based on the isobologram analysis (Fig. 2D), we estimated the highest synergistic effect at $50 \%$ response for these concentrations. For each experimental group, six replicates were analyzed by LC-MS/MS. Label-free proteome analysis based on precursor ion intensities revealed quantitative data for 2689 protein groups. After excluding proteins quantified with only one unique peptide, 1839 proteins remained for further analysis (Table S1, ESI $\dagger$ ). Proteins passing significance and fold change criteria $\left(p<0.05, \log _{2}\right.$ ratio (treated/ untreated) $>1$ or $<-1$ ) were defined as significantly regulated. Visualizations of the proteomics data as volcano plots as well as the number of up- and down-regulated proteins, respectively, are shown in Fig. 3. The treatment with the drug combination of MLN4924 and cisplatin induced the highest number of protein regulations (223 proteins). This was expected, since the drug combination exhibits a $50 \%$ cytotoxic effect at the used concentration, which is not the case in the treatments with the individual drugs. However, even if marginally cytotoxic at the used concentration, both individual compounds induced 57 (cisplatin) and 163 (MLN4924) significant protein regulations.

\section{Synergistic proteome alterations}

To investigate whether the aforementioned synergistic effect also manifests on the proteome level, the data set obtained in the quantitative proteome analysis were further systematically analyzed. Therefore, the lists of proteins significantly two-fold regulated by the individual drugs and the respective combination were compared based on protein accessions numbers. This comparison revealed 93 proteins whose regulation passed both fold change and significance criteria exclusively in the presence of the drug combination. This number corresponds to $42 \%$ of all proteins regulated under the respective conditions (223 proteins) (Fig. 4A). However, the presence of particular proteins in the respective complement of a Venn diagram is not necessarily indicative of a synergistic regulation. Instead, this observation

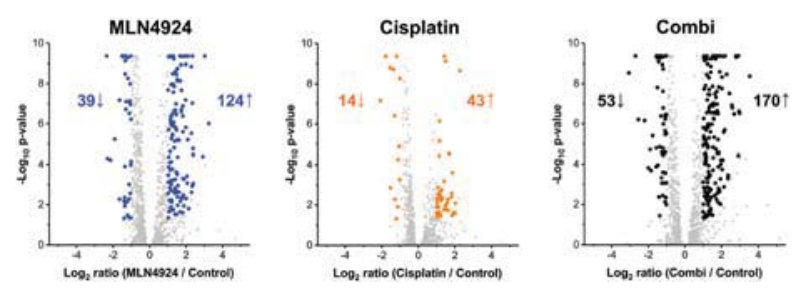

Fig. 3 Results of the quantitative proteome analysis visualized as volcano plots (proteins quantified with at least two unique peptides). Proteins with significantly altered abundances (two-fold change of expression, $p<0.05$ ) are highlighted as colored dots. Numbers of up- and down-regulated proteins are given in each plot as well.


Fig. 4 Comparative analysis of proteomics data. (A) shows a Venn diagram derived from an accession-based comparison of significantly regulated proteins (two-fold change of expression, $p<0.05$ ). (B) shows box plots of regulation profiles of the 93 proteins exclusively found in the complement of the combined treatment. Here, the same trends towards up- and down-regulation of the single drugs are observable. Data points represent $\log _{2}$ ratio values of individual proteins. Boxes represent 25 th and 75th percentiles and whiskers indicate the standard deviation. (C) shows a plot of synergistic scores of 93 proteins exclusively found in the complement of the combined treatment. The dashed line represents the threshold of $>2$ used to highlight synergistically regulated proteins. (D) shows the regulation profile of SUB1. Columns represent averaged normalized protein abundances as determined by mass spectrometry. Data points indicate protein abundances determined in the individual replicates and error bars the standard deviation. (E) shows verification of the synergistic regulation of SUB1 by western blotting. GAPDH shows equal sample loading.

only shows that these proteins exhibit at least a significant two-fold regulation by the treatment with the drug combination, but not the individual drugs. This could simply rely on additive rather than synergistic effects, especially if one considers the fact that the same regulation trends were observable for most of the proteins (Fig. 4B). Actually, in samples treated with the individual drugs, for most of these 93 proteins the same trend towards up- or down-regulation was observable, but to a lower extent.

To cope with this issue we calculated synergistic scores to highlight proteins, whose abundance changes are not merely consequences of additive effects, namely summed regulations induced by the individual drugs. To do so, we defined a synergistic score (eqn (1)) implying an effect-based strategy relying on a response additivity model, which in contrast to a dose-effect-based strategy does not require knowledge of drug concentrations exhibiting a particular effect. ${ }^{24}$

Synergistic score

$$
=\frac{\log _{2}\left(\text { ratio }\left(\frac{\text { combi }}{\text { untreated }}\right)\right)}{\log _{2}\left(\text { ratio }\left(\frac{\text { MLN4924 }}{\text { untreated }}\right)\right)+\log _{2}\left(\text { ratio }\left(\frac{\text { cisplatin }}{\text { untreated }}\right)\right)}
$$

Here, for each protein, abundance changes under each experimental condition relative to the untreated control are considered. 
Synergistic scores $>1$ indicate proteins, which are stronger regulated in the combination as compared to the sum of regulations caused by the individual compounds. Based on the strength of superadditive regulation as indicated by the synergistic score, one can highlight synergistically regulated proteins. In contrast, scores $<1$ indicate proteins whose abundance changes are lower in the drug combination as compared to the individual components. For such proteins, the regulation in the drug combination is rather subadditive and therefore not synergistic. In the case of the 93 proteins previously highlighted by the accession-based comparison, only 41 proteins exhibited synergistic scores $>1$ (between 1 and 12), whereas for the other 52 proteins scores between 1 and 0.57 were found. A ranked plot of the synergistic scores of the 93 proteins is shown in Fig. 4C.

To shed light on proteins exhibiting comparatively strong synergistic regulations, the 93 proteins were ranked regarding their synergistic scores. A threshold of $>2$ was applied to consider the fact that fold changes used to calculate the synergistic scores have particular imprecisions, which depend on the accuracy of the applied quantitative approach. In summary, five proteins were identified, which are synergistically regulated upon treatment with a combination of MLN4924 and cisplatin, namely activated RNA polymerase II transcriptional coactivator p15 (SUB1), Nucleoredoxin (NXN), Glutamate-cysteine ligase regulatory subunit (GCLM), Hemoglobin subunit alpha (HBA1), and Secretory carrier-associated membrane protein 3 (SCAMP3). A complete list of the 93 proteins including regulation factors upon different treatments and the respective synergistic scores is provided in Table S2 (ESI $\dagger$ ).

For further analysis, we focused on the protein with the highest synergistic score, namely SUB1. Here, the strong synergistic regulation as indicated by a synergistic score of 12 also nicely shows up in the regulation profile based on protein abundances in the different experimental groups (Fig. 4D). Furthermore, the mass spectrometry-based results were verified by immunoblotting (Fig. 4E), where an intense band for SUB1 could only be detected after combined treatment.

DNA damage, oxidative stress and apoptosis in the synergistic drug combination

Activated RNA polymerase II transcriptional coactivator p15 (SUB1), also known as Positive cofactor 4 (PC4) is a DNA-binding protein and highly conserved among several species. ${ }^{25}$ Apart from its initially prescribed role as a transcriptional coactivator, several other functions have been discovered over the years, such as roles in maintaining genome stability by protecting DNA against oxidative stress and participating in DNA repair. For example, it has been demonstrated that SUB1/PC4 acts as an activator of nonhomologous end joining (NHEJ) and DNA double-strand break (DSB) repair activity. ${ }^{26}$ Additionally, accumulation of SUB1/PC4 at DNA damage sites has been shown using live cell microscopy ${ }^{27}$ and a critical role in DNA damage repair re-routing has been proposed. ${ }^{28}$ Furthermore, increased expression of SUB1/PC4 induced by oxidative stress has been shown and a protective role of SUB1/PC4 against oxidative DNA damage upon hydrogen peroxide treatment has been elucidated in SUB1-deficient yeast models. ${ }^{29,30}$ Moreover, SUB1/PC4 has been described as an activator of the tumor suppressor protein p53 and consequently p53-mediated apoptosis. ${ }^{31,32}$ Since SUB1/PC4 is p53-responsive at the same time, a positive feedback loop for p53 regulation has been proposed. ${ }^{33}$ Taking together these reports, SUB1/PC4 plays an important role in the response to DNA damage and oxidative stress as well as in the induction of p53-mediated apoptosis. Consequently, considering the synergistic up-regulation of SUB1/PC4, one can assume that the combination of cisplatin and MLN4924 increases oxidative stress and DNA damage and thereby induces the synergistic up-regulation of SUB1/PC4, which in turn can lead to increased apoptosis via the p53-mediated apoptotic pathway. In fact, such a cooperative effect of MLN4924 and cisplatin in terms of increased DNA damage and oxidative stress is not unexpected, since several reported studies already proposed respective modes of action. For example, on the one hand, increased DNA damage and oxidative stress has been observed as a result of combination treatment in various ovarian cancer cell lines. ${ }^{14}$ On the other hand, increased apoptosis and DNA damage have been shown in the high-grade bladder urothelial carcinoma cell lines NTUB1 and T24 treated with combinations of cisplatin and MLN4924. ${ }^{10}$ Hence, we analyzed DNA damage and apoptosis via western blot analysis of known marker proteins as well as oxidative stress via fluorescence-based ROS assay. As expected, the apoptosis markers active caspase 3 and cleaved PARP were found more abundant in the drug combination as compared to the individual compounds. Furthermore, an increased abundance of the DNA-damage marker $\gamma$-H2AX highlighted increased DNA damage after combined treatment (Fig. 5A). Despite not being significant, the conducted ROS assay displayed distinct trends towards increased abundance of cellular ROS after combined treatment (Fig. 5B), which is in line with the fact that proteins associated with oxidative stress such as NRX, ${ }^{34}$ HBA1, ${ }^{17,35}$ and GCLM $^{36}$ were also found as synergistically regulated proteins.
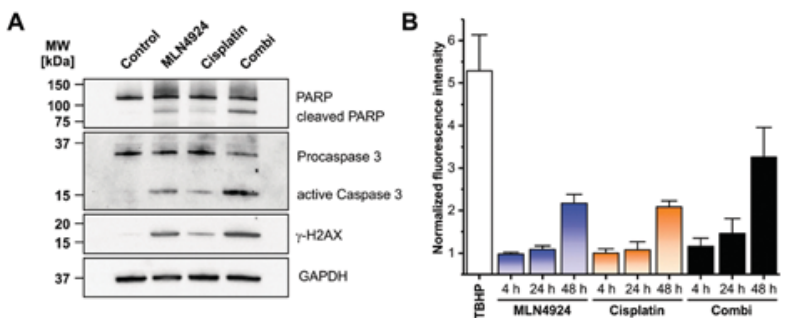

Fig. 5 Analysis of DNA damage, oxidative stress and apoptosis. (A) shows western blot results of marker proteins for DNA damage $(\gamma-\mathrm{H} 2 \mathrm{AX})$ and apoptosis (PARP and caspase 3). GAPDH served as a loading control. (B) Shows the results of fluorescence-based measurement of ROS generation depending on time and type of treatment using $2^{\prime}, 7^{\prime}$-dichlorofluorescin diacetate (DCFDA). Measurements were performed at least as triplicates. Values (mean values \pm standard error of mean) were normalized to untreated controls. As positive control, cells were treated with tert-butyl hydroperoxide (TBHP) (200 $\mu \mathrm{M}, 60 \mathrm{~min}$ treatment). 


\section{Experimental}

\section{Cells, media and compounds}

HCT-116 cells (p53 wild type) were obtained from Leibniz Institute DSMZ-German Collection of Microorganisms and Cell Cultures (Braunschweig, Germany) and were grown in a humidified incubator with $5 \% \mathrm{CO}_{2}$ at $37{ }^{\circ} \mathrm{C}$ in DMEM medium supplemented with 10\% fetal calf serum. MLN4924 was purchased from Active Biochem (Cat\#: A-1139) and cisplatin was provided by Prof. Dr Bernhard Lippert (Fakultät Chemie und Chemische Biologie (CCB), Technische Universität Dortmund, Germany).

\section{Cell viability assays}

Cell viability was measured using the XTT (2,3-bis-(2-methoxy-4nitro-5-sulfophenyl)-2 $H$-tetrazolium-5-carboxanilide) assay (Thermo Fisher Scientific, Rockford, IL, USA) in a 96-well plate format based on a previously reported protocol. ${ }^{17}$ Briefly, a number of 5000 cells in $200 \mu \mathrm{l}$ medium were grown per well for $24 \mathrm{~h}$ at $37^{\circ} \mathrm{C}$. To screen various drug combinations in a 96-well plate format, the treatment was conducted with dilution series of the individual compounds and respective combinations. Therefore, $100 \mu \mathrm{l}$ of medium was removed and $50 \mu \mathrm{l}$ medium containing various amounts of cisplatin was added. Afterwards, $50 \mu \mathrm{l}$ medium containing different concentrations of MLN4924 was added as well. By doing so, in each row of the plate the following concentrations of cisplatin were adjusted: $0 \mu \mathrm{M}, 1.03 \mu \mathrm{M}, 2.06 \mu \mathrm{M}, 4.12 \mu \mathrm{M}$, $8.3 \mu \mathrm{M}, 16.5 \mu \mathrm{M}, 33 \mu \mathrm{M}$ and $66 \mu \mathrm{M}$. In turn, the different columns of the plate contained the following concentrations of MLN4924: $0 \mu \mathrm{M}, 0.03125 \mu \mathrm{M}, 0.0625 \mu \mathrm{M}, 0.125 \mu \mathrm{M}, 0.25 \mu \mathrm{M}$, $0.5 \mu \mathrm{M}, 1 \mu \mathrm{M}, 2 \mu \mathrm{M}, 4 \mu \mathrm{M}, 8 \mu \mathrm{M}$. As a result, a $10 \times 8$ matrix containing all possible combinations of the abovementioned drug concentrations was generated. The remaining wells of the plate were used for carrier controls (DMSO 1\%) and blanks. After incubation for $48 \mathrm{~h}$ at $37{ }^{\circ} \mathrm{C}, 100 \mu \mathrm{l}$ medium was removed and $50 \mu \mathrm{l}$ of a freshly prepared XTT reagent solution in medium (1 $\mathrm{mg} \mathrm{ml}^{-1}$ XTT, $25 \mu \mathrm{M}$ phenazine methosulfate) was added to each well. After incubation for an additional $2 \mathrm{~h}\left(37^{\circ} \mathrm{C}\right)$, absorbance was measured on a Tecan Infinite M200 Pro plate reader at $450 \mathrm{~nm}$ (normalized to $650 \mathrm{~nm}$ ). Cell viability was calculated relative to the absorbance of carrier controls. Average values were obtained by performing three independent cell viability assays. Dose-response curves were generated by sigmoidal fits using the DoseResp function implemented in the Origin Pro 2017 software (OriginLab Corporation, Northhampton, MA, USA), yielding EC $_{50}$ values of cisplatin in the absence and presence of various concentrations of MLN4924 and vice versa. For isobologram analysis, ${ }^{22}$ drug combinations leading to a $50 \%$ reduction of cell viability were plotted. Data points located on the $y$ - and $x$-axis, respectively, represent $\mathrm{EC}_{50}$ values of single drug treatments. A further synergy analysis was conducted using SynergyFinder (https://synergyfinder.fimm.fi). ${ }^{23}$

\section{Sample preparation for proteomics experiments}

Samples ( $n=6$ for each condition) for quantitative proteome analyses were prepared in a 6-well plate format. A number of 100000 cells in $2.5 \mathrm{ml}$ medium were seeded per well and incubated at $37{ }^{\circ} \mathrm{C}$ for $24 \mathrm{~h}$. Then, the medium was exchanged by a fresh medium containing the desired drug concentrations: cisplatin $(5 \mu \mathrm{M})$, MLN4924 $(0.2 \mu \mathrm{M})$, a combination of both drugs, and DMSO (1\%) as a carrier control. The treatment was carried out for $48 \mathrm{~h}$ at $37{ }^{\circ} \mathrm{C}$. Afterwards, cells were harvested using a cell scraper and the cell suspension was transferred into a $15 \mathrm{ml}$ reaction tube followed by centrifugation at $4{ }^{\circ} \mathrm{C}$ (100 rcf, $10 \mathrm{~min}$ ). The supernatant was removed and the cell pellet was resuspended in $1 \mathrm{ml}$ PBS and transferred into a $1.5 \mathrm{ml}$ tube. After centrifugation $\left(100 \mathrm{rcf}, 4{ }^{\circ} \mathrm{C}, 10 \mathrm{~min}\right)$ and removal of the supernatant, the pellet was washed twice by resuspending in $1 \mathrm{ml}$ cold PBS and subsequent centrifugation (100 ref, $4{ }^{\circ} \mathrm{C}, 10 \mathrm{~min}$ ). The resulting cell pellet was lysed in $40 \mu \mathrm{l}$ lysis buffer ( $50 \mathrm{mM} \mathrm{NH}_{4} \mathrm{HCO}_{3}, 0.1 \%$ Rapigest) by sonication on ice for $5 \mathrm{~min}$. Afterwards, cell debris was removed by centrifugation (16000 rcf, $4{ }^{\circ} \mathrm{C}, 50 \mathrm{~min}$ ) and the supernatant (approx. $30 \mu \mathrm{l}$ ) was transferred into a fresh $0.5 \mathrm{ml}$ reaction tube. The protein concentration of the supernatant was determined by Bradford assay. For in-solution digestion, an amount of $4 \mu \mathrm{g}$ protein was adjusted to a total volume of $20 \mu \mathrm{l}$ with $50 \mathrm{mM}$ $\mathrm{NH}_{4} \mathrm{HCO}_{3}$, reduced using $20 \mathrm{mM}$ dithiothreitol for $30 \mathrm{~min}$ at $60{ }^{\circ} \mathrm{C}$, and alkylated with $15 \mathrm{mM}$ iodoacetamide for $30 \mathrm{~min}$ at ambient temperature. Trypsin was added $\left(3 \mu \mathrm{l}, 0.1 \mu \mathrm{g} \mu \mathrm{l}^{-1}\right)$ for digestion for $16 \mathrm{~h}$ at $37{ }^{\circ} \mathrm{C}$. Digestion was stopped by acidification with $1.5 \mu \mathrm{l}$ of $10 \%$ trifluoroacetic acid (TFA) for $30 \mathrm{~min}$ at $37{ }^{\circ} \mathrm{C}$. Insoluble hydrolyzed surfactant was removed by centrifugation (16000 rcf, $4{ }^{\circ} \mathrm{C}, 10 \mathrm{~min}$ ). The collected supernatant was separated into aliquots containing $350 \mathrm{ng}$ tryptic peptides. These were dried in a centrifugal evaporator, reconstituted in $17 \mu \mathrm{l} 0.1 \%$ TFA, and stored at $-80{ }^{\circ} \mathrm{C}$ until LC-MS/MS analysis.

\section{LC-MS/MS analysis}

LC-MS/MS analyses were carried out on an Ultimate 3000 RSLCnano liquid chromatography system online coupled with an Orbitrap Elite mass spectrometer (both Thermo Fisher Scientific). In each run, $309 \mathrm{ng}$ tryptic peptides in $15 \mu \mathrm{l} 0.1 \%$ TFA were injected. The peptides were pre-concentrated for $7 \mathrm{~min}$ on a trap column (Acclaim ${ }^{R}$ PepMap 100, $75 \mu \mathrm{m} \times 2 \mathrm{~cm}, \mathrm{C} 18,5 \mu \mathrm{m}, 100 \AA$ ) using $30 \mu \mathrm{min}^{-1} 0.1 \%$ TFA as an eluting solvent. Subsequent separation on an analytical column (Acclaim ${ }^{\circledR}$ PepMap RSLC, $75 \mu \mathrm{m} \times 50 \mathrm{~cm}$, nano Viper, C18, $5 \mu \mathrm{m}, 100 \AA$ ) was carried out using a gradient from 5 to $40 \%$ solvent B in solvent A over $98 \mathrm{~min}$ (solvent A: $0.1 \%$ formic acid; solvent B: $0.1 \%$ formic acid, $84 \%$


oven temperature of $60{ }^{\circ} \mathrm{C}$. The mass spectrometric analysis was conducted in a data-dependent acquisition mode. Full scans were acquired in the Orbitrap analyzer (mass range: $300-2000 \mathrm{~m} / \mathrm{z}$, resolution: 60000) in a data-dependent mode. The 20 most abundant ions of a spectrum acquired at the MS1 level were fragmented by collision-induced dissociation (normalized collision energy: $35 \%$, isolation width: $2 \mathrm{~m} / \mathrm{z}$ ) and measured in the linear ion trap.

\section{Protein identification and quantification}

Peptide and protein identification was carried out using the Proteome Discoverer software (ver. 1.4, Thermo Fisher Scientific Inc.). 
The human UniProt/Swiss-Prot database (release 2015_11, number of sequences: 20194) was searched using Mascot (ver. 2.5.1, Matrix Science Ltd, London, UK). Mass tolerances were set to $5 \mathrm{ppm}$ and 0.4 Da for precursor and fragment ion masses, respectively. Trypsin was set as protease with one allowed missed cleavage. Variable oxidation of methionine was considered and carbamidomethylation of cysteine was set as static modification. Confidence of peptide identifications was estimated using the target decoy PSM validator function implemented in Proteome Discoverer software. Only high confident peptide identifications with false discovery rates $<1 \%$ were considered in the analysis. Protein grouping function was disabled. Lists of peptide spectrum matches and corresponding peptide and protein identifications were exported as Excel sheets for subsequent import into the quantification software.

Peptide identifications were mapped with respective LC-MS features, which were quantified in Progenesis QI for Proteomics (ver. 2.0.5, Nonlinear Dynamics Ltd, Newcastle upon Tyne, UK). A detailed description of the quantification procedure was reported earlier. ${ }^{37,38}$ Briefly, all LC-MS/MS runs were matched to a reference run of a master mix consisting of equal amounts of all samples. From all selected features, only those comprising charges of $2+$ to $4+$ were selected. Features with two or less isotopic peaks were discarded. Afterwards, raw abundances of each feature were automatically normalized for correcting experimental variations. The samples were grouped corresponding to the experimental conditions and quantified features were mapped to peptide identification obtained from the abovementioned database searches. Protein grouping was disabled and protein quantification was conducted based on unique peptides only. The generated list of quantified proteins was exported for subsequent statistical analysis.

The mass spectrometry proteomics data have been deposited to the ProteomeXchange Consortium via the PRIDE ${ }^{39}$ partner repository with the dataset identifier PXD009185 and 10.6019/PXD009185. Data were uploaded using the ProteomeXchange Submission Tool (ver. 2.3.2). ProCon-PROteomics CONversion tool (ver. 0.9.641) was used for the necessary conversion of Proteome Discoverer result files into the mzIdentML standard format. ${ }^{40}$ An overview of uploaded LC-MS/MS runs including sample identifiers is provided as Table S3 (ESI $\dagger$ ).

\section{Statistical analysis}

Statistical analysis of the label-free data was conducted with an in-house developed R script (R Foundation for Statistical Computing, Vienna, Austria) as described earlier. ${ }^{41}$ Briefly, normalized intensities of all quantified proteins were arcsinhyp-transformed and analyzed by means of one-way ANOVA. The generated $p$-values were FDR-adjusted according to Benjamini and Hochberg. ${ }^{42}$ For proteins with FDR-adjusted $p$-values $<0.05$, a post hoc test for direct comparison of treatment conditions was conducted. Therefore, Tukey's Honest Significant Difference method (THSD) was used. Statistical analysis of cell viability experiments was performed using the nonparametric Mann-Whitney $U$ test with Origin Pro 2017 software (OriginLab Corporation, Northhampton, MA, USA).

\section{Western blots}

Selected proteins were analyzed by means of western blotting following a previously reported protocol. ${ }^{37}$ The following primary and secondary antibodies were used: Anti-PARP (rabbit, Abcam ab191217), Anti-Histone H2A.X (rabbit, Abcam ab81299), AntiGAPDH (mouse, Proteintech 60004-1-Ig); Anti-SUB1 (rabbit, Atlas HPA001311); Anti-Caspase 3, (rabbit, Abcam ab13847), Peroxidase AffiniPure Goat Anti-Rabbit IgG (H + L) (Jackson Immunoresearch 111-035-144), Peroxidase AffiniPure Goat Anti-Mouse IgG (H + L) (Jackson Immunoresearch 115-035-062).

\section{Reactive oxygen species assay}

The formation of reactive oxygen species (ROS) was analyzed using $2^{\prime}, 7^{\prime}$-dichlorofluorescin diacetate (DCFDA, Sigma Aldrich) assay in a black-walled 96-well plate. Therefore, 15000 cells were seeded per well and grown for $24 \mathrm{~h}$ in $50 \mu \mathrm{l}$ medium (without phenol red). The medium was removed, cells were washed once with PBS and incubated for 30 min with $100 \mu$ DCFDA solution (20 $\mu \mathrm{M}$ in PBS). After removing the labeling solution, cells were washed with PBS and $100 \mu \mathrm{l}$ medium containing the desired drug concentrations (0.2 $\mu \mathrm{M}$ MLN4924, $5 \mu \mathrm{M}$ cisplatin, $0.2 \mu \mathrm{M}$ MLN4924 $+5 \mu \mathrm{M}$ cisplatin) was added. Medium containing no supplements, DMSO $(1 \%)$ or tert-butyl hydroperoxide $(200 \mu \mathrm{M})$ was used as the carrier and positive control, respectively. At given time points, fluorescence was measured using a Tecan Infinite M200 Pro plate reader (excitation wavelength $485 \mathrm{~nm}$, emission wavelength $535 \mathrm{~nm}$ ). Each experiment was performed at least in triplicate with twelve individual measurements per well.

\section{Conclusions}

In the reported study we investigated the synergistic interaction of the widely used chemotherapeutic drug cisplatin with the neddylation inhibitor MLN4924 (pevonedistat) in the human colon carcinoma cell line HCT-116. By using cell viability assays and isobologram analyses we were able to reproduce the reported synergy of these compounds. To investigate if and to what extent the synergistic interaction manifests on the proteome level in terms of synergistic protein regulations, a quantitative proteome analysis using an ion-intensity-based approach was conducted. Here, proteome modulations of individual drugs and the drug combination were assessed and synergistic scores for protein regulations were calculated based on a response additivity model. Following this approach, we were able to identify activated RNA polymerase II transcriptional coactivator p15 (SUB1) also known as Positive cofactor 4 (PC4) as a highly synergistically up-regulated protein. This result was further verified by western blotting. Based on literature data and the finding that DNA damage, oxidative stress and apoptosis were found to be increased in the drug combination, we propose that the synergistic drug combination of cisplatin and MLN4924 induces increased SUB1/PC4 expression in response to oxidative stress and DNA damage, which in turn can lead to increased apoptosis in a p53-dependent manner. However, future studies are needed to shed more light on the 
functional roles of SUB1/PC4 as well as other synergistically regulated proteins in the investigated drug combination, for example, by respective gain and loss of function experiments or the investigation of p53-deficient cells. Apart from that, the reported study demonstrated the feasibility of quantitative proteomics to investigate modes of action of drug combinations with synergistic effects by highlighting proteins likely associated with such effects using a synergistic scoring approach. Furthermore, in a more clinical context, the knowledge about such proteins can help to nominate novel targets for specific combination treatments, which potentially could increase treatment efficiency and reduce undesired side effects at the same time.

\section{Conflicts of interest}

There are no conflicts to declare.

\section{Acknowledgements}

The authors gratefully acknowledge financial support received from the Deutsche Forschungsgemeinschaft (TRR60 Z3) and within PURE (Protein research Unit Ruhr within Europe) (233-1.08.03.03031-68079), a project of NRW, a federal state of Germany. We thank Prof. Dr Bernhard Lippert for providing cisplatin. Furthermore, we thank Kristin Rosowski and Stefanie Tautges for their excellent technical support.

\section{Notes and references}

1 B. Rosenberg, L. VanCamp, J. E. Trosko and V. H. Mansour, Nature, 1969, 222, 385.

2 B. W. Harper, A. M. Krause-Heuer, M. P. Grant, M. Manohar, K. B. Garbutcheon-Singh and J. R. Aldrich-Wright, Chemistry, 2010, 16, 7064.

3 S. Dasari and P. B. Tchounwou, Eur. J. Pharmacol., 2014, 740, 364 .

4 L. Kubiczkova, L. Pour, L. Sedlarikova, R. Hajek and S. Sevcikova, J. Cell. Mol. Med., 2014, 18, 947.

5 L. J. Crawford and A. E. Irvine, Blood Rev., 2013, 27, 297.

6 T. A. Soucy, P. G. Smith, M. A. Milhollen, A. J. Berger, J. M. Gavin, S. Adhikari, J. E. Brownell, K. E. Burke, D. P. Cardin, S. Critchley, C. A. Cullis, A. Doucette, J. J. Garnsey, J. L. Gaulin, R. E. Gershman, A. R. Lublinsky, A. McDonald, H. Mizutani, U. Narayanan, E. J. Olhava, S. Peluso, M. Rezaei, M. D. Sintchak, T. Talreja, M. P. Thomas, T. Traore, S. Vyskocil, G. S. Weatherhead, J. Yu, J. Zhang, L. R. Dick, C. F. Claiborne, M. Rolfe, J. B. Bolen and S. P. Langston, Nature, 2009, 458, 732.

7 J. E. Brownell, M. D. Sintchak, J. M. Gavin, H. Liao, F. J. Bruzzese, N. J. Bump, T. A. Soucy, M. A. Milhollen, X. Yang, A. L. Burkhardt, J. Ma, H. K. Loke, T. Lingaraj, D. Wu, K. B. Hamman, J. J. Spelman, C. A. Cullis, S. P. Langston, S. Vyskocil, T. B. Sells, W. D. Mallender, I. Visiers, P. Li, C. F. Claiborne, M. Rolfe, J. B. Bolen and L. R. Dick, Mol. Cell, 2010, 37, 102.
8 L. Zhou, W. Zhang, Y. Sun and L. Jia, Cell. Signalling, 2018, 44, 92.

9 V. T. Le-Trilling, D. A. Megger, B. Katschinski, C. D. Landsberg, M. U. Ruckborn, S. Tao, A. Krawczyk, W. Bayer, I. Drexler, M. Tenbusch, B. Sitek and M. Trilling, Sci. Rep., 2016, 6, 19977.

10 I. L. Ho, K. L. Kuo, S. H. Liu, H. C. Chang, J. T. Hsieh, J. T. Wu, C. K. Chiang, W. C. Lin, Y. C. Tsai, C. T. Chou, C. H. Hsu, Y. S. Pu, C. S. Shi and K. H. Huang, Sci. Rep., 2015, 5, 16948.

11 A. A. Jazaeri, E. Shibata, J. Park, J. L. Bryant, M. R. Conaway, S. C. Modesitt, P. G. Smith, M. A. Milhollen, A. J. Berger and A. Dutta, Mol. Cancer Ther., 2013, 12, 1958.

12 W. C. Lin, K. L. Kuo, C. S. Shi, J. T. Wu, J. T. Hsieh, H. C. Chang, S. M. Liao, C. T. Chou, C. K. Chiang, W. S. Chiu, T. Y. Chiu, Y. S. Pu, I. L. Ho, Z. H. Wang, S. C. Chang, S. H. Liu, Y. M. Jeng and K. H. Huang, Am. J. Cancer Res., 2015, 5, 3350.

13 S. Lin, Z. Shang, S. Li, P. Gao, Y. Zhang, S. Hou, P. Qin, Z. Dong, T. Hu and P. Chen, Oncol. Lett., 2018, 15, 2583.

14 S. T. Nawrocki, K. R. Kelly, P. G. Smith, C. M. Espitia, A. Possemato, S. A. Beausoleil, M. Milhollen, S. Blakemore, M. Thomas, A. Berger and J. S. Carew, Clin. Cancer Res., 2013, 19, 3577.

15 K. Garcia, J. L. Blank, D. C. Bouck, X. J. Liu, D. S. Sappal, G. Hather, K. Cosmopoulos, M. P. Thomas, M. Kuranda, M. D. Pickard, R. Liu, S. Bandi, P. G. Smith and E. S. Lightcap, Mol. Cancer Ther., 2014, 13, 1625.

16 D. A. Megger, J. Philipp, V. T. K. Le-Trilling, B. Sitek and M. Trilling, Front. Immunol., 2017, 8, 1139.

17 D. A. Megger, K. Rosowski, C. Radunsky, J. Kosters, B. Sitek and J. Muller., Dalton Trans., 2017, 46, 4759.

18 V. Calamia, J. Mateos, P. Fernandez-Puente, L. Lourido, B. Rocha, C. Fernandez-Costa, E. Montell, J. Verges, C. Ruiz-Romero and F. J. Blanco, Sci. Rep., 2014, 4, 5069.

19 X. Liu, P. J. Pai, W. Zhang, Y. Hu, X. Dong, P. Y. Qian, D. Chen and H. Lam, Sci. Rep., 2016, 6, 19841.

20 L. Bianchi, F. Bruzzese, A. Leone, A. Gagliardi, M. Puglia, E. D. Gennaro, M. Rocco, A. Gimigliano, B. Pucci, A. Armini, L. Bini and A. Budillon, Proteomics, 2011, 11, 3725.

21 J. I. Toth, L. Yang, R. Dahl and M. D. Petroski, Cell Rep., 2012, 1, 309.

22 R. J. Tallarida, J. Pharmacol. Exp. Ther., 2006, 319, 1.

23 A. Ianevski, L. He, T. Aittokallio and J. Tang, Bioinformatics, 2017, 33, 241.

24 J. Foucquier and M. Guedj, Pharmacol. Res. Perspect., 2015, 3, e00149.

25 M. Garavis and O. Calvo, Curr. Genet., 2017, 63, 1023.

26 K. Batta, M. Yokokawa, K. Takeyasu and T. K. Kundu, J. Mol. Biol., 2009, 385, 788.

27 O. Mortusewicz, W. Roth, N. Li, M. C. Cardoso, M. Meisterernst and H. Leonhardt, J. Cell Biol., 2008, 183, 769.

28 R. B. Caldwell, H. Braselmann, U. Schoetz, S. Heuer, H. Scherthan and H. Zitzelsberger, Sci. Rep., 2016, 6, 28890.

29 J. Y. Wang, A. H. Sarker, P. K. Cooper and M. R. Volkert, Mol. Cell. Biol., 2004, 24, 6084. 
30 L. Yu, H. Ma, X. Ji and M. R. Volkert, Mol. Cell. Biochem., 2016, 412, 165.

31 K. Batta and T. K. Kundu, Mol. Cell. Biol., 2007, 27, 7603.

32 S. Banerjee, B. R. Kumar and T. K. Kundu, Mol. Cell. Biol., 2004, 24, 2052.

33 A. H. Kishore, K. Batta, C. Das, S. Agarwal and T. K. Kundu, Biochem. J., 2007, 406, 437.

34 S. Kneeshaw, R. Keyani, V. Delorme-Hinoux, L. Imrie, G. J. Loake, T. Le Bihan, J. P. Reichheld and S. H. Spoel, Proc. Natl. Acad. Sci. U. S. A., 2017, 114, 8414.

35 W. Liu, S. S. Baker, R. D. Baker, N. J. Nowak and L. Zhu, PLoS One, 2011, 6, e24363.

36 C. M. Krejsa, C. C. Franklin, C. C. White, J. A. Ledbetter, G. L. Schieven and T. J. Kavanagh, J. Biol. Chem., 2010, 285, 16116.

37 D. A. Megger, T. Bracht, M. Kohl, M. Ahrens, W. Naboulsi, F. Weber, A. C. Hoffmann, C. Stephan, K. Kuhlmann,
M. Eisenacher, J. F. Schlaak, H. A. Baba, H. E. Meyer and B. Sitek., Mol. Cell. Proteomics, 2013, 12, 2006.

38 J. Padden, D. A. Megger, T. Bracht, H. Reis, M. Ahrens, M. Kohl, M. Eisenacher, J. F. Schlaak, A. E. Canbay, F. Weber, A. C. Hoffmann, K. Kuhlmann, H. E. Meyer, H. A. Baba and B. Sitek, Mol. Cell. Proteomics, 2014, 13, 2661. 39 J. A. Vizcaino, A. Csordas, N. del-Toro, J. A. Dianes, J. Griss, I. Lavidas, G. Mayer, Y. Perez-Riverol, F. Reisinger, T. Ternent, Q. W. Xu, R. Wang and H. Hermjakob, Nucleic Acids Res., 2016, 44, D447.

40 G. Mayer, C. Stephan, H. E. Meyer, M. Kohl, K. Marcus and M. Eisenacher, J. Proteomics, 2015, 129, 56.

41 W. Naboulsi, T. Bracht, D. A. Megger, H. Reis, M. Ahrens, M. Turewicz, M. Eisenacher, S. Tautges, A. E. Canbay, H. E. Meyer, F. Weber, H. A. Baba and B. Sitek, Biochim. Biophys. Acta, 2016, 1864, 1579.

42 Y. Benjamini and Y. Hochberg, J. R. Stat. Soc. Series B Stat. Methodol., 1995, 57, 289. 